
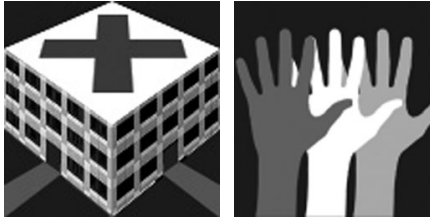

\title{
"It's been an Experience, a Life Learning Experience": A Qualitative Study of Hospitalized Patients with Substance Use Disorders
}

\author{
Christine M. Velez, MSW' ${ }^{1,2}$, Christina Nicolaidis, MD, MPH ${ }^{2,3}, P$. Todd Korthuis, MD, MPH ${ }^{3}$, and \\ Honora Englander, $M D^{1,4}$ \\ ${ }^{1}$ Clinical Integration, Oregon Health \& Science University, Portland, OR, USA; ${ }^{2}$ School of Social Work, Portland State University, Portland, OR, USA; \\ ${ }^{3}$ Division of General Internal Medicine, Oregon Health \& Science University, Portland, OR, USA; ${ }^{4}$ Division of Hospital Medicine, Oregon Health \& \\ Science University, Portland, OR, USA.
}

BACKGROUND: Individuals with substance use disorders (SUD) have high rates of chronic illness and readmission, yet few are engaged in addiction treatment. Hospitalization may be a reachable moment for initiating and coordinating addiction care, but little is known about motivation for change in the inpatient setting.

OBJECTIVE: To explore the experiences of hospitalized adults with SUD and to better understand patient and system level factors impacting readiness for change.

DESIGN: We performed a qualitative study using individual interviews. The study was nested within a larger mixed-methods needs assessment.

PARTICIPANTS AND SETTING: Hospitalized adults admitted to medical or surgical units at an urban academic medical center who reported high-risk alcohol or drug use on AUDIT-C or single-item drug use screener.

APPROACH: We conducted a thematic analysis, using an inductive approach at a semantic level.

KEY RESULTS: Thirty-two patients participated. The mean age was 43 years; $75 \%$ were men, and 68\% identified as white. Participants reported moderate to high-risk alcohol (39\%), amphetamine (46\%), and opioid (65\%) use. Emergent themes highlight the influence of hospitalization at the patient, provider, and health system levels. Many patients experienced hospitalization as a wake-up call, where mortality was motivation for change and hospitalization disrupted substance use. However, many participants voiced complex narratives of social chaos, trauma, homelessness, and chronic pain. Participants valued providers who understood SUD and the importance of treatment choice. Patient experience suggests the importance of peers in the hospital setting, access to medication-assisted treatment, and coordinated care post-discharge.

CONCLUSIONS: This study supports that hospitalization offers an opportunity to initiate and coordinate addiction care, and provides insights into patient, provider, and health system factors which can leverage the reachability of this moment.

KEY WORDS: qualitative research; substance-related disorders; opioidrelated disorders; motivation; hospital; health reform.

Received June 27, 2016

Revised October 13, 2016

Accepted October 26, 2016

Published online December 12, 2016
$\mathrm{J}$ Gen Intern Med 32(3):296-303

DOI: $10.1007 / \mathrm{s} 11606-016-3919-4$

(c) Society of General Internal Medicine 2016

\section{INTRODUCTION}

Individuals with substance use disorders (SUD) have high rates of chronic illness, hospitalization, and readmission. ${ }^{1-5}$ Despite frequent contact with healthcare systems, many people are not engaged in addiction treatment. ${ }^{3,6}$ Hospitalization is often unplanned, and most persons do not come to the hospital seeking addiction treatment. However, hospitalization may be an opportunity for healthcare providers to initiate treatment for substance use. There is limited empirical evidence regarding motivating factors for treatment and readiness to change during an acute medical hospitalization. ${ }^{3}$

Motivation for change is nuanced and dynamic. ${ }^{3}$ Few studies have explored factors associated with readiness to change among hospitalized adults with SUD. Pollini et al. found that a confluence of internal ("tired of using") and external (probation, family) factors were motivation for treatment, and that individuals who believed their medical condition would worsen as a result of continued use were twice as likely to be motivated to change. ${ }^{3}$ However, little is known about hospitalized patients' treatment preferences, or about the systemlevel factors that might support their engagement in SUD treatment in the hospital.

A handful of interventions have shown that it is feasible to initiate medication-assisted treatment (MAT) for SUD in the hospital, ${ }^{4,7,8}$ and that doing so can increase the likelihood of patients engaging in SUD treatment after discharge. However, these interventions have shown high rates of attrition in treatment. Understanding patient perspectives on SUD treatment options - including MAT_might improve the outcomes of these interventions. We contend that the patient's voice is critical in shaping system improvements and best practices.

Our study explores the experiences of patients with SUD who were hospitalized for medical problems, in order to better understand patient- and system-level factors that impact readiness to change and to inform future interventions. 


\section{METHODS}

We conducted in-person interviews with hospitalized adults who reported high-risk alcohol or drug use on the AUDIT-C and/or single-item drug use screening tool. ${ }^{9,10}$ All protocols and materials were approved by the institutional review board of Oregon Health \& Science University.

\section{Setting and Study Design}

This qualitative study was nested within a larger mixed-methods needs assessment of 185 hospitalized adults at an urban academic teaching hospital in Portland, Oregon. Oregon is an Accountable Care Act (ACA) Medicaid expansion state. In 2014, 60\% of adult inpatients at our hospital resided in the Portland metro area. Most of the remaining were from surrounding rural areas. Fifty-three percent of adult inpatients had public insurance (Medicare and/or Medicaid). The objective of the needs assessment was to inform a multi-component intervention for hospitalized adults with SUD, which we will describe elsewhere. The full research team, which includes three physicians and one social worker, has diverse experience in SUD.

\section{Participants}

Our intent was to obtain rich, qualitative data from a wide range of participants. All participants completed an in-person survey assessing their substance use. After survey completion, we invited some participants to complete a semi-structured audiorecorded interview. All participants were English-speaking adults between the ages of 18 and 65 years, who were hospitalized on a general medicine or surgery ward. We excluded patients admitted to intensive care or psychiatric units, incarcerated individuals, and those who were too obtunded to participate. We intentionally sampled patients who were medically stable and could engage in a longer, in-depth conversation.

\section{Data Collection}

We interviewed all participants in their hospital rooms using a semi-structured interview guide created by the full team (Appendix, available online). All participants gave verbal consent and were compensated with a $\$ 5$ gift card. One author (CV), a social worker, conducted interviews from September 2014 to April 2015. Interviews lasted from 15 to $45 \mathrm{~min}$. The analysis team met halfway through data collection to review transcripts, and modified the interview guide to improve the depth and richness of the data. We ended recruitment after reaching theme saturation. Recordings were transcribed, de-identified, and transferred to ATLAS.ti (version 7.5.9; Scientific Software Development $\mathrm{GmbH}$, Berlin, Germany) for data analysis and retrieval.

\section{Data Analysis}

The interdisciplinary team conducted a thematic analysis ${ }^{11}$ using an inductive approach at the semantic level. The coding team comprised two general internists ( $\mathrm{HE}$ and $\mathrm{CN}$ ) and one social worker $(\mathrm{CV})$. Using an iterative process, we selected a preliminary coding schema, independently coded transcripts, and then met as a group or in dyads to discuss and reconcile codes, identify emergent themes, and resolve discrepancies through consensus. All coders (HE, CN, CV) coded the initial seven transcripts to establish a preliminary coding schema; coders then coded the remaining transcripts in dyads. One coder (CV) coded all transcripts; the other two coders ( $\mathrm{HE}$ and $\mathrm{CN}$ ) divided the transcripts evenly.

\section{RESULTS}

Participant characteristics are summarized in Table 1. Thirtytwo hospitalized adults participated in our study. The mean age was 43 years; $75 \%$ were men, and $68 \%$ identified as white. Participants reported moderate- to high-risk alcohol (39\%), amphetamine (46\%), and opioid (65\%) use, indicating substantial polysubstance use across the sample. All participants had health insurance. Qualitative results are presented below as themes and sub-themes. Seventeen participants are repre-

Table 1 Participant Characteristics

\begin{tabular}{|c|c|}
\hline Characteristic & $n(\%)$ \\
\hline Mean age, years (SD) & $43(10.4)$ \\
\hline Male sex & $24(75 \%)$ \\
\hline \multicolumn{2}{|l|}{ Race/ethnicity } \\
\hline White & $21(66 \%)$ \\
\hline Native American & $4(13 \%)$ \\
\hline African American & $1(3 \%)$ \\
\hline Hispanic & $5(16 \%)$ \\
\hline Multiracial/other & $4(8 \%)$ \\
\hline \multicolumn{2}{|l|}{ Housing } \\
\hline Own/rent & $14(44 \%)$ \\
\hline Staying with friends or family & $5(16 \%)$ \\
\hline Shelter/streets/car/motel & $11(34 \%)$ \\
\hline Nursing home/rehab facility & $2(6 \%)$ \\
\hline \multicolumn{2}{|l|}{ Education } \\
\hline Grades 1-12/GED & $15(47 \%)$ \\
\hline College $1-4+$ years & $17(53 \%)$ \\
\hline \multicolumn{2}{|l|}{ Annual household income } \\
\hline$\$ 10,000$ & $15(42 \%)$ \\
\hline$\$ 10,000-20,000$ & $5(15 \%)$ \\
\hline$\$ 20,000-50,000$ & $3(10 \%)$ \\
\hline Refused & $9(29 \%)$ \\
\hline \multicolumn{2}{|l|}{ Marital status } \\
\hline Married or living with long-term partner & $3(9 \%)$ \\
\hline Widowed/divorced/separated & $14(44 \%)$ \\
\hline Never married & $15(47 \%)$ \\
\hline \multicolumn{2}{|l|}{ Moderate- to high-risk use by substance* } \\
\hline Alcohol & $12(39 \%)$ \\
\hline Amphetamines & $12(46 \%)$ \\
\hline Opioids & $13(65 \%)$ \\
\hline Health insurance-yes & $32(100 \%)$ \\
\hline Veterans Administration benefits & $2(6 \%)$ \\
\hline Medicare & $4(13 \%)$ \\
\hline Medicaid & $26(81 \%)$ \\
\hline \multicolumn{2}{|l|}{ Diagnosis category } \\
\hline Alcoholic pancreatitis/GI bleed & $2(6 \%)$ \\
\hline Alcohol withdrawal & $4(13 \%)$ \\
\hline Cardiovascular disease (e.g. heart failure) & $2(6 \%)$ \\
\hline Cellulitis/abscess/wound infection & $8(25 \%)$ \\
\hline Endocarditis/bacteremia & $4(13 \%)$ \\
\hline Osteomyelitis & $2(6 \%)$ \\
\hline Pulmonary disease (e.g. respiratory failure) & $5(16 \%)$ \\
\hline Trauma/fracture & $2(6 \%)$ \\
\hline Other & $3(9 \%)$ \\
\hline
\end{tabular}

* Moderate to high risk use as assessed by the alcohol, smoking and substance use involvement screening test (ASSIST) ${ }^{22}$ 
sented in the following quotations. Emergent themes affecting readiness to change as described by participants are best categorized as relating to patient, provider, and system-level factors.

\section{Patient-Level Factors}

Hospitalization Serves as a Wake-Up Call: Mortality is Motivating. Participants described hospitalization as a wake-up call that was motivation for change. For some, near-death experiences highlighted the potential of not being present as parents to their children or to experience key life events. For others, the reality that active substance use increased health risks for needed medical treatment was a powerful incentive. One participant stated:

"I wish I didn't put myself in the predicament of coming to the hospital so much. It's been an experience, a life learning experience. Coming here so much has helped me notice and realize to wake up. It's time to stop doing what I'm doing, to get to the sober and clean path."

Many patients described near-death experiences, and that facing mortality was motivation for recovery. As one woman stated:

"I knew it was to that point where I almost died the other day, and I have a 3-year-old little boy, and it's not where I want to be."

Illness severity was motivation for change. Some patients described having been told by providers that active substance use precluded certain treatment options because it increased the risk of complications. As one patient in need of hernia repair described, "The doctor said, 'We can't fix you until you stop using heroin."”

Hospitalization Interrupts Drug Use. Acute hospitalization interrupts drug use and may create a window of opportunity for change. As one patient stated:

"While I'm in the hospital, there's no managing the drug use, because it's being managed in the sense that I am not using and I'm being cared for physically."

Hospitalization presents an abrupt change in environment, often forcing detoxification or sobriety, limiting patients' access to substances, creating a reprieve from negative peer influences, and interrupting physical and behavioral patterns of SUD.

Overpowering Compulsion to Use. Participants expressed an overpowering compulsion to use despite hospitalization, mortality, or potential efforts to engage in treatment. Many described feeling powerless over their addiction, feeling that no hospital intervention could help them begin a path to recovery. One participant stated:

"My whole addiction to heroin-it's going to end up killing me, and I still know that I'm going to go back out there and do it, and that's just so f***ed up... it's cost me everything and I still do it. Someday it will cost me my life."

Pain Complicates Care. Participants perceived pain as a barrier to quitting. This was especially true among people with opioid use disorders. One participant explained:

"I can't even think about getting my drug use under control until I figure out how to get my pain managed and my health concerns under control."

Others described the complex interplay of pain management and SUD, and how a failure to address pain increased the risk for continued use and for leaving the hospital against medical advice. Many participants described substance use as a way to self-medicate and manage pain. As one participant noted:

"Not being in intolerable pain, that's a pretty big deal, 'cause when my pain gets really bad, that's when I want to leave and go use, because it hurts, because a lot of people don't want to give you pain management because you are an addict."

\section{Provider-Level Factors}

Importance of Choice. Participants emphasized the importance of having choices regarding their care, and not feeling coerced into accepting substance use treatment. For example, one patient stated:
"When you tell me what to do, I'm a mule. I dig my hooves in and I'm like, uh-uh [shaking head], I make my own decisions. But if I have somebody to talk to that could understand where I'm coming from, yeah, I could see that helping people."

Participants recognized the value of patient-centered treatment options. As one participant explained:

"Regardless of the approach, providers need to recognize where the person is, give the person a choice, and provide the best care possible."

Provider Understanding of SUD is Important. Many participants felt that hospital providers lacked an understanding of SUD. Participants described feeling that providers didn't understand how daunting recovery efforts 
might be, and that it was not a simple choice to just say no. As one participant noted:

"Most of us that do it can't stand it. I hate the stuff...it is wretched...it's like damned if you do, damned if you don't... when I do it I don't even feel good anymore, like it takes so much just to be okay, to be normal. It's like when I use I just feel normal...so they don't understand that."

Importance of Caring and Non-Judgmental Staff. Participants emphasized the importance of caring, nonjudgmental staff who understood addiction. Caring staff supported participants staying in the hospital instead of leaving against medical advice. As one participant said:

"One of the reasons I've stayed at this hospital is because they have been very flexible and understanding of me. And not just that, but a few of them are actual ex-addicts themselves, so they can understand what I'm going through, because I don't feel judged. I feel welcome when people are understanding that this is more of a disease than a personal choice. It's a lot easier for me to make better decisions and feel good about what I'm doing...."

Many described feeling stigmatized in hospitals, and perceptions that providers viewed substance use as a moral failing. One participant stated:

"In the other hospitals, people are so judging, you know, and think that you're a heroin addict, so you are just like, you know, a bad person."

Participants described ambivalence that might change over the course of hospitalization. Participants perceived providers who understood ambivalence as a natural part of substance use and recovery as supportive. A non-judgmental approach facilitated patient engagement in treatment. One participant described her experience with a provider who reached out several times:

"At first I didn't want talk to the social worker or a drug and alcohol doctor. But to just pop in like Dr. [name of physician] popped in...[showed me that] he actually really cared, because even though [people with SUD] are saying no, they may not be wanting to say no... I was embarrassed, and I didn't want to have to ask for the resources or the help."

Importance of Effective Withdrawal Management. Fear of withdrawal dominated many participants' experience during hospitalization, and highlighted that adequate withdrawal management is critical to any engagement in care. As one participant described:

"It's not just a fear; it's that you become physically, like, crippled and sick from the withdrawal of opiates and methamphetamines. Diarrhea, vomiting, sweats, chills - it's just like the flu times ten. I would rather go through childbirth, honestly, than withdrawing. So it's not that I want to get high; it's that I have to. It's become a necessity, or I'm dying. It feels like I'm dying."

Participants described variation in how providers treated withdrawal. As one patient explained, "Every doctor has different protocols..." Participants noted that untreated withdrawal led to suffering, leaving the hospital against medical advice, and feeling that care was inadequate.

\section{Health System-Level Factors}

Hospitalization Does Not Address Life Stressors and

Trauma. Participants voiced complex narratives about life stressors and trauma impacting their use. One patient stated, "All I've seen is death and carnage my whole life." This sentiment was echoed by another patient who described:

"I've just been exposed to so much just pain and hardship and just mean, awful people, that having someone nice around is extremely important to me."

As hospitalization focuses on acute medical needs, it often does not focus on people's complex mental health needs that may impact their care and their substance use.

Hospitalization Does Not Address Basic Needs. Finally, some participants felt that efforts to improve addiction care in the hospital would be pointless, because hospitalization does not address their basic needs. Patients lacked housing and "basic gear" like backpacks, sleeping bags, and food. One participant described hospital detoxification, saying:

"I almost see it like being futile, because I don't know how I'm going to do when I get back out there, because I have nowhere to go and nothing. I don't even have a jacket at this point, sleeping bag-I don't even have that now."

Value of Peers in the Hospital Setting. Participants stated that talking to peers-people with lived experience in recovery-helped to build trust and credibility. As one person commented:

"I don't like to talk to people, like counselors and people like that haven't used drugs....who try to tell you how to feel or think about drugs." 
Participants felt that hospital-based peers would provide valuable support and highlight the possibility for recovery. As one participant described:

"It's having that extra support-most every counselor I've met has been a former addict, and there couldn't be a better sponsor than somebody that's been there and done it, and has worked through it, and is doing it daily."

Access to Medication-Assisted Therapy (MAT). Many participants were interested in starting MAT in the hospital and felt that it improved the quality of care. As one woman noted:

"When I went to [another hospital], they did not have doctors who are allowed to prescribe that [suboxone] and I was out of luck. So then, here, I found [you] have a doctor who could prescribe me methadone or suboxone. That's awesome. It may not be for everybody."

Other participants were unfamiliar with MAT, especially for treatment of alcohol use disorders. Some participants raised concerns about side effects of MAT and how it might affect their medical conditions. Others viewed MAT as a "crutch" or substitute for their substance of choice, and voiced fears that withdrawal from MAT was more painful than from other substances. As one person stated:

"I've heard that the addiction to methadone is a lot worse than heroin; suboxone and buprenorphine are totally different."

Some participants voiced a strong preference for buprenorphine over methadone for treatment of opioid use disorder. Some did not want the structure of daily dosing, and others described past negative experiences. As one patient stated:

"Suboxone allows me to function much better than methadone. With methadone, I'd be so tired that it would make me crave stimulants. I mean, I almost need to do crystal meth with it."

Need for Coordinated, Timely Care After Discharge. Participants who underwent detoxification in the hospital recognized that prolonged time to treatment after discharge presented a significant barrier. Otherwise, "they throw you back out to the same old thing." This was particularly true as participants reflected on the possibility of starting methadone or other MAT in the hospital:

"It sucks to get out there if you're given a gap where you don't have any [methadone] - if I leave here and I don't have meds, I'm f***ed. I'm not more likely to use, I am-period, end of story — going to use; I'm going straight to the man."

Participants discussed needing coordinated care that went beyond a list of resources. One participant explained that being given a list of resources was unhelpful and felt dismissive:

"I don't like hospitals because they don't help - they put a strict line on. [They think] 'I'll bet she's not gonna get help, she's a heroin addict, this is what we see in all addicts. Just put her on the list, whatever, with everybody else... give her the list of resources, if she wants help she'll figure it out.' I've had bad experiences at other hospitals; they feel it's useless to waste their time when all I want is help."

Participants also noted that assessment alone in the hospital — without access to treatment or referrals - felt futile:

"The questions that they ask, 'Ihow much do you smoke? how much do you drink? and how much medical drugs do you use?' I know they have to use that all for the medical end of it, but I'm not really sure where they intertwine with everything else at this point."

\section{DISCUSSION}

Our study uses patient perspectives to identify patient, provider, and health system-level factors that may impact patients' readiness to change and to engage in SUD care during hospitalization. Hospitalization served as a wake-up call, forcing increased awareness of mortality, the harmful effects of SUD, and the costs of SUD on parenting and other relationships, while also temporarily disrupting drug use.

Many participants indicated high interest in treatment, including MAT and a desire to work with peers. While many highlighted the benefits of initiating treatment in the hospital, others felt that barriers such as homelessness, trauma, and pain would limit their ability to engage in SUD care after discharge.

Participants emphasized the importance of non-judgmental and caring staff as a key factor in engendering their trust and engagement. Participants also emphasized the importance of choice, both to avoid feeling pressured or coerced into treatment, and with regard to the array of treatment options available during and after hospitalization. Participants voiced a need for improved care coordination and more timely transition from inpatient to outpatient SUD treatment.

Our findings parallel earlier results, such as those from Pollini et al., which indicate that a confluence of internal and external factors influences readiness to change. Pollini et al. found that "fear of illness," "physical health concerns," and "family issues" were factors motivating change. ${ }^{3}$ Our study 
builds on these findings and further explores system-level factors that can influence readiness for and engagement in treatment among hospitalized adults with SUD.

Our study identified several key factors influencing engagement in the hospital. First, participants identified the presence of caring staff as a key contributor to their staying in the hospital instead of leaving against medical advice, and in engaging in treatment. Our findings echo those in studies of patients with SUD in primary care, which revealed higher satisfaction among patients who perceived that providers trusted, respected, and empathized with them, ${ }^{12,13}$ as well as higher satisfaction for patients who perceived physician concerns about "you as a patient" and "being treated like a patient instead of a drug addict." ${ }^{14}$ Feeling cared for in a relationship was particularly important to vulnerable hospitalized patients. Participants also noted the importance of staff who knew how to treat withdrawal, ${ }^{15}$ and felt that variation in treatment implied that some providers were delivering substandard care. This mirrors the results of Merrill et al., ${ }^{16}$ who found that physicians lacked a standard approach to commonly encountered clinical issues experienced by hospitalized patients with SUD, especially the assessment and treatment of pain and opiate withdrawal. Over a decade later, our findings show a continued need for protocols and staff training to improve provider competence in identifying, assessing, and treating SUD in the hospital.

Our study builds upon existing work showing that it is feasible to initiate MAT among hospitalized adults, including treatment for opioid use disorder with methadone $e^{4,17}$ and buprenorphine, ${ }^{7}$ and treatment for alcohol use disorder with naltrexone. ${ }^{8}$ Our findings suggest that when MAT is offered in the hospital, it is important to provide an array of treatment options, and that MAT "may not be for everybody." These findings support literature reports of patients wanting an individualized approach to treatment. ${ }^{18}$

However, while earlier studies have supported the feasibility of initiating MAT in the hospital, ${ }^{4,7,8}$ our study highlights key barriers that could limit the generalizability of these interventions. Specifically, the gap between discharge and entry into treatment can be substantial, and systems need rapid-access pathways from inpatient hospitalization to community SUD treatment. Our study also highlights the need for more patient education about MAT (which is treatment, and not a "crutch"), as well as staff education, and highlights patients' limited knowledge about medications for alcohol use disorder.

Limitations of our study include that all patients were insured, and that it was conducted at a single site, which may limit generalizability. The depth of interviews was sometimes limited due to the challenges of conducting research during an acute hospitalization; however, obtaining information in real time was critical to understanding the patient experience. Racial and ethnic diversity was low, so findings may not be transferable to more diverse populations. Finally, our study assessed only patient perspectives. Data from providers and other stakeholders may further enrich our understanding of issues affecting the care of hospitalized patients with SUD.

Our study has several key implications. First, our findings underscore the need for additional training for physicians, nurses, social workers, and others around SUD. ${ }^{19}$ Training should focus on understanding SUD as a chronic illness, recognition and treatment of withdrawal, and knowledge of MAT. In addition, it is particularly important for social workers to understand that providing a list of resources without other support can feel dismissive to some patients and may weaken the therapeutic relationship.

Many participants felt that they were stigmatized in hospital settings due to SUD. There is an urgent need to promote a cultural shift away from thinking of SUD as a moral failing, to understanding it as a treatable chronic disease. In particular, our findings suggest that provider awareness of ambivalence towards treatment and a continued use of trauma-informed, person-centered approaches are critical in caring for hospitalized adults with SUD.

Our findings have specific implications for hospital systems looking to initiate SUD for inpatients. Our results underscore the importance of making MAT more accessible in the inpatient environment and in the community, and the importance of partnerships and pathways to close the time gap between hospital discharge and community care.

Our study also illustrates the effect of greater societal factors such as poverty and homelessness, which cannot be addressed through hospitalization alone. ${ }^{20,21}$ Policies that expand access to SUD treatment and address social determinants of health should be part of an integrated approach to improving care for hospitalized adults with SUD.

These findings have informed a multi-component hospital based-intervention aimed at improving care for people with SUD. This includes the creation of a hospitalbased inpatient addiction consulting service comprising board-certified addiction medicine physicians, a social worker with extensive training in SUD, and peer recovery mentors. It also includes rapid-access pathways to community SUD care and innovative models of SUD care for medically complex adults, including increased access to MAT. Future research will assess the effectiveness of this program in improving outcomes.

Our study shows that hospitalization is a reachable moment for initiating and coordinating addiction care, and it identifies key factors at the provider, health system, and societal levels that can improve patients' experience and engagement in treatment. Future studies could explore patients' experience after discharge, and evaluate interventions aimed at addressing these key system-level factors. 
Acknowledgments: This work was funded by Oregon Health \& Science University. We would like to thank Sarann Bielavitz for her contributions as a project coordinator for this study. This paper was presented at the annual Society for Social Work and Research conference in January 2016 in Washington, DC.

Corresponding Author: Honora Englander, MD; Oregon Health \& Science University, 3181 SW Sam Jackson Park Road, Portland, OR 97239, USA (e-mail: englandh@ohsu.edu).

\section{Compliance with Ethical Standards:}

Conflict of Interest: The authors declare that they have no conflict of interest.

\section{APPENDIX: INTERVIEW GUIDE}

1. Most people who use <drugs or alcohol $>$ have mixed feeling about continuing to use/drink. Tell me a little more about your thoughts on your <alcohol/ drug $>$ use.

a. Tell me a little more about what you have done in the past to reduce or stop using.

b. What got in the way?

c. What did you find most helpful?

d. If you could do it over again, what would you do differently?

e. What do you think would have been able to make things better?

Now what do you think would be most useful to you?

2. I'd like to hear a little more about your experiences during this hospitalization. Please tell me what it's been like to be in the hospital.

a. How has being in the hospital affected your thoughts about your drug use?

b. What's been helpful to support recovery?

c. What's gotten in the way?

d. What would be helpful to you?

3. I would also like to hear about your experiences with social workers during this hospitalization.

a. Have you seen a social worker here? If so, what has that been like?

b. If not, did you want to see a social worker?

c. What was helpful? What could have been done better?

4. Reflecting back, how do you think your substance use/ alcohol use affects your use of medical services such as ER visits, hospitalizations, or primary care?

a. What types of things keep you from going to primary care?

b. What would help you need to go to ED less frequently?

c. What, if anything, do you think could have helped you stay out of the hospital?

5. Tell me about what you think will be most important in terms of managing your alcohol/drug use after you leave the hospital? a. What would that look like?

b. What might get in the way?

c. What could we do while you are in the hospital to make that happen?

6. One idea is to have drug and alcohol counselors meet with patients while they are in the hospital. What do you think about that?

a. What would be helpful?

b. Can you think of anything that would be a problem?

7. Another idea is to have an addiction medicine physician available who could prescribe medications while patients are still in the hospital. For example, they can start people who use opioids on medications like methadone or buprenorphine. Or they could start people who use alcohol on medications such as naltrexone. What do you think about that idea?

a. Why would you be interested or not interested in something like that?

b. What would make it more likely for you to take such a medication? What would keep you from taking such a medication?

8. Another idea is that we could have peers come into the hospital to offer support and help connect with treatment. Peers would be people who are in recovery themselves. What do you think of that idea?

a. What would be helpful?

b. What problems might there be?

c. What would make you comfortable talking with a peer?

\section{REFERENCES}

1. O'Toole TP, Pollini RA, Ford DE, Bigelow G. The effect of integrated medical-substance abuse treatment during an acute illness on subsequent health services utilization. Med Care. 2007;45(11):1110-5.

2. Pecorano A, Horton T, Ewen E, et al. Early data from project engage: a program to identify and transition medically hospitalized patients into addictions treatment. Addict Sci Clin Pract. 2012;7(20):1-7.

3. Pollini RA, O'Toole TP, Ford D, Bigelow G. Does this patient really want treatment? Factors associated with baseline readiness to change among hospitalized substance using adults interested in treatment. Addict Behav. 2006;31:1904-18.

4. Shanahan CW, Beers D, Alford DP, Brigandi E, Samet JH. A transitional opioid program to engage hospitalized drug users. J Gen Intern Med. 2010;25(8):803-8.

5. Walley AY, Paasche-Orlow M, Lee EC, et al. Acute care utilization among medical inpatients discharged with a substance use disorder. J Addict Med. 2012;6(1):50-6.

6. Haber PS, Demirkol A, Lange K, Murnion B. Management of injecting drug users admitted to hospital. Lancet. 2009;374:1284-93.

7. Liebschutz JM, Crooks D, Herman D, et al. Buprenorphine treatment for hospitalized opioid-dependent patients: a randomized clinical trial. JAMA Intern Med. 2014;174:1369-76.

8. Wei J, Defries T, Lozada M, Young N, Huen W, Tulsky J. An inpatient treatment and discharge planning protocol for alcohol and dependence: efficacy in reducing 30-day readmissions and emergency department visits. J Gen Intern Med. 2014;30:365-70.

9. World Health Organization (WHO). Screening and brief intervention for hazardous and harmful alcohol use. 2012. Available at: http://www.who. int/mental_health/mhgap/evidence/alcohol/q1/en/. Accessed October 26, 2016. 
10. Smith $\mathbf{P}$, Schmidt S, Allensworth-Davies D, Saitz R. A single-question screening test for drug use in primary care. Arch Intern Med. 2010;170:1155-60.

11. Braun V, Clarke V. Using thematic analysis in psychology. Qual Res Psychol. 2006;3:77-101.

12. Korthuis PT, Gregg J, Rogers WE, McCarty D, Nicolaidis C, Boverman J. Patients' reasons for choosing office-based buprenorphine: preference for patient-centered care. J Addict Med. 2010;4:204-10.

13. Hall M. Measuring patients' trust in their primary care providers. Med Care Res Rev. 2002;59:293-318.

14. Barry DT, Moore BA, Pantalon MV, et al. Patient satisfaction with primary care office-based buprenorphine/naloxone treatment. J Gen Intern Med. 2007;22:242-5.

15. Milloy E. Withdrawal from methadone in US prisons: cruel and unusual? Lancet. 2015;386:316-8.

16. Merrill JO, Rhodes LA, Deyo RA, Marlatt A, Bradley KA. Mutual mistrust in the medical care of drug users: the keys to the "narc" cabinet. J Gen Intern Med. 2002; 17:327-33.
17. Ti L, Ti L. Leaving the hospital against medical advice among people who use illicit drugs: a systematic review. Am J Public Health. 2015; 105:53-9.

18. Institute of Medicine. Improving the quality of health care for mental and substance-use conditions: quality chasm series. Washington, DC: National Academies Press; 2006.

19. Wakeman SE, Baggett MV, Pham-Kanter G, Campbell EG. Internal medicine residents' training in substance use disorders: a survey of the quality of instruction and residents' self perceived preparedness to diagnose and treat addiction. Subst Abus. 2013;34:363-70.

20. Sun AP. Helping homeless individuals with co-occurring disorders: the four components. Soc Work. 2012;57:23-37.

21. Upshur CC, Weinreb L, Cheng DM, Kim TW, Samet JH, Saitz R. Does experiencing homelessness affect women's motivation to change alcohol or drug use? Am J Addict. 2014;23:76-83.

22. WHO ASSIST Working Group. The alcohol, smoking and substance use involvement screening test (ASSIST): development, reliability and feasibility. Addiction. 2002;97:1183-94. 